\title{
ON THE DERIVATION OF ABUNDANCES BY MEANS OF FEATURES IN THE SEQUENCES OF THE OLD OPEN STAR CLUSTERS
}

\author{
A. MAEDER \\ Observatoire de Genève, Switzerland
}

\begin{abstract}
Stellar evolution near the main-sequence is still subject to many discussions. In addition to the case of the cluster M 67 (Racine, 1971), quite systematic differences have been encountered during the comparison of theoretical isochrones and observed sequences in the colour-magnitude diagram of the old open star clusters (Maeder, 1974). In the case of M 67 and NGC 188, many attempts to discuss the characteristics of their sequences (such as the gap corresponding to the hydrogen-exhaustion phase) in terms of chemical composition have been made (e.g. Aizenman et al., 1969; Demarque and Schlesinger, 1969; Demarque and Heasley, 1971; Torres-Peimbert, 1971; Hejlesen et al., 1972; Caloi et al., 1974); some of these works have suggested to explain the gap parameters by a high metal content. Attemps to explain the differences between models and observations by means of simplified models with overshooting from convective cores have also been made (Maeder, 1973; Prather and Demarque, 1974). Let us also note that it has been shown that it is unlikely that the anomalies found are due to systematic effects, like the interstellar reddening, binarity, rotation or effects in calibrations or composition of the initial homogeneous models.
\end{abstract}

We now turn to the interior models, which are challenged by the above anomalies. It is clear that the evolutionary tracks depend closely on the $X$-profiles in the stellar interiors; these profiles are determined themselves mainly by the size of the convective cores during the evolution. In the usual sets of models of stellar interiors, convection in the cores is not treated explicitly. There are well-known reasons for that (Biermann, 1932), which are certainly fully justified as far as the course of the various structural variables is concerned, but certainly not as far as the extent of the convective zone is concerned. Thus, a new method has been developed (Maeder, 1975) allowing to perform an explicit treatment of convection in the cores with non-local expressions of the mixing-length formalism. An iterative process is used to integrate simultaneously the equations of stellar structure and the equations describing convection.

The application of the method to stars in the range of 1.25 to $3 M_{\odot}$ shows that the zone fully mixed by turbulent convective motions extends appreciably farther from the limit given by Schwarzschild's criterion. The overshooting, which amounts to about $14 \%$ of a mixing-length for the homogeneous initial models, brings only negligible modifications in the results of the initial models. Thus, the location of the zero-age sequence in the HRD remains unmodified by the overshooting from the cores. However, the consequences of this effect are much larger during evolution and several changes occur in the

B. Hauck and P. C. Keenan (eds), Abundance Effects in Classification, 97-99. All Rights Reserved. Copyright $\odot 1976$ by the IAU. 


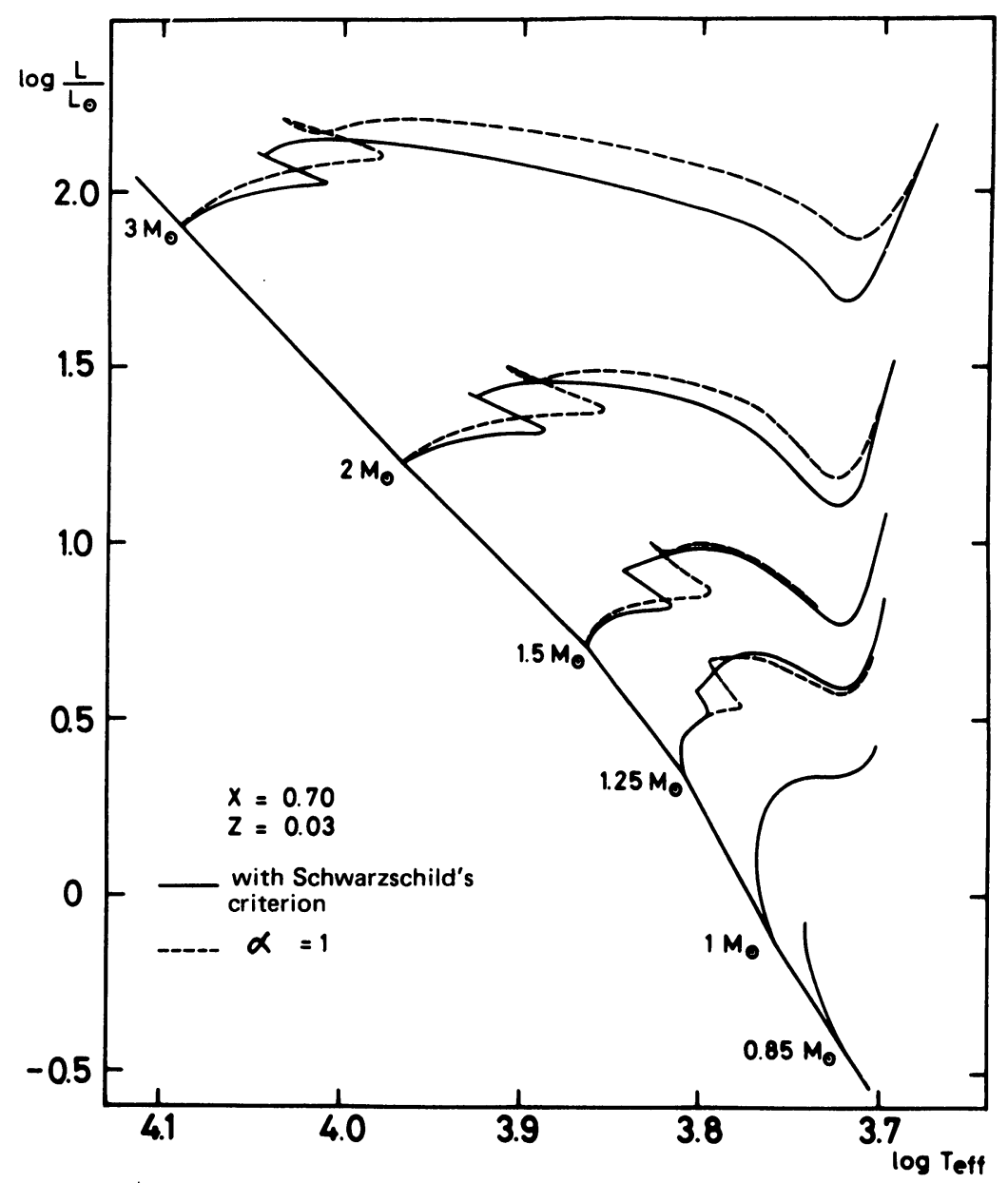

Fig. 1. Evolutionary paths in the theoretical HRD for stars with local theories of convection in the core (Schwarzschild's criterion) and with non-local theories predicting an overshooting from the core. The case, where $\alpha=l / H_{p}=1$ in the core is shown. The case $\alpha=\frac{1}{2}$ has also been computed. The overshooting also modifies the lifetimes appreciably.

shape of the tracks and in the lifetimes. Figure 1 shows some results; the case where $\alpha=l / H_{p}=1$ in the core is illustrated. The case $\alpha=\frac{1}{2}$ has also been computed. In models with overshooting, the hook in the evolutionary tracks is described very rapidly, so that such models predict that the visible gap will occur almost vertically in the HRD. Actual comparisons show that models with a mild overshooting $\left(\alpha=\frac{1}{2}\right.$, i.e. the zone of overshooting contains about $2-3 \%$ of $M_{r} / M$ at $1.5 M_{\odot}$ ) provides simultaneously for all features of the sequences in the HRD a very satisfactory agreement, which is never obtained with the usual grid of models.

From the work, very briefly outlined above, we may make the following remarks concerning the determinations of chemical abundances by means of parameters of cluster 
sequences: (1). It is necessary to consider in the comparisons of models and observations all the features describing the sequence of a cluster in the region of the gap and not only to consider one or two gap parameters. (2). The case of M 67 and NGC 188 cannot be disconnected from the fact that also for other slightly younger clusters, a very poor agreement is realized between observations and models. (3). The determination of $X, Y$ and $Z$ content by means of gap parameters is rather hazardous, as these parameters are very sensitive to the process of overshooting which is hydrodynamically possible at the edge of the core. (4). More reliance must certainly be given to the determinations of helium and metal content, which are based on the location of the zero-age sequence, $(c f$. Morton and Adams, 1968), as this location is very independent on the extent of the fully mixed zone in the centres of upper main-sequence stars.

\section{References}

Aizenman, M. L., Demarque, P., and Miller, R. H.: 1969, Astrophys. J. 155, 973.

Biermann, L.: 1932, Z. Astrophys. 5, 117.

Caloi, V., Castellani, V., and Di Paolo, N.: 1974, Astron. Astrophys. 30, 349.

Demarque, P., Heasley, J. N.: 1971, Astrophys. J. 163, 547.

Demarque, P., Schlesinger, B. M.: 1969, Astrophys. J. 155, 965.

Hejlesen, P. M., Jorgensen, H. E., Petersen, J. O., and Romcke, L.: 1972, in G. Cayrel de Strobel and A. M. Delplace (eds.), IAU Colloquium. 17, Observatoire de Paris.

Maeder, A.: 1973, in Stellar Instability and Evolution, IAU Symposium No 59, p. 109.

Maeder, A.: 1974, Astron. Astrophys. 32, 177.

Maeder, A.: 1975, Astron. Astrophys. 40, 303.

Morton, D. C., Adams, T. F.: 1968, Astrophys. J. 151, 611.

Prather, M. J., Demarque, P.: 1974, Astrophys. J. 193, 109.

Racine, R.: 1971, Astrophys. J. 168, 393.

Torres-Peimbert, S.: 1971, Bol. Obs. Tonantzintla Tacubaya 6, 3.

\section{DISCUSSION}

Spinrad: Do your new 'overshoot' convective core models change the ages of the old galactic clusters?

Maeder: The overshooting from the convective core mainly redistributes the lifetimes among the various evolutionary phases. However, the age estimates will probably be increased by about $20 \%$.

Bell: Could you tell us how much an isochrone will differ, if it is computed with and without allowing for overshooting?

Maeder: The differences in the isochrone produced by the overshooting depend on the part of the isochrone you are considering. For example, a very large effect exists for the height of the top of the gap above the zero-age sequence. This feature may differ by about $0^{m} \cdot 8$, when overshooting is included. Quantities, usually called $\Delta M_{\text {bol }}$ (gap) and $\Delta M_{\text {bol }}$ (peak) are also modified by the overshooting from convective core. 